\title{
Experiments on the Growth of Fungi on Culture Media.
}

\author{
BY
}

WILLIAM BROWN, M.A., D.SC.

(From the Department of Plant Physiology and Pathology, Imperial College of Science and

\section{Technology, London.) \\ With seven Figures in the Text.}

THE experiments to be described in the present paper had their origin 1 in certain unexplained results which were met with in the course of an investigation of the growth of fungi in atmospheres of different composition. ${ }^{1}$ It was there found that the effect of moderate concentrations of carbon dioxide (10-20 per cent.) consisted in general in a reduction of growth as measured in terms of the diameter of the fungal colony. This result was obtained, for example, with cultures of Botrytis cinerea and Alternaria grossulariae on a variety of media. Here the cultures in air were in advance of those in to per cent. carbon dioxide throughout the whole period of examination. On the other hand, certain clearly marked exceptions to this rule came to light. Thus it was found that with cultures of Sphaeropsis malorum on certain media the colony growing in to per cent. carbon dioxide at first lagged behind the one growing in air, but later on very distinctly surpassed it. It was with the view to a better understanding of such behaviour that the present study was undertaken.

In the course of this study it was found that the method of experiment adopted (which consisted chiefly in the systematic measurement of the rate of spread of the fungal colony) appeared to offer a promising basis for physiological work on fungi, on which account the scope of the investigation has been considerably extended. Briefly expressed, the aim of the investigation has developed into the study of the form of fungal cultures, chiefly in its relation to external factors. In how far this can be accomplished with the methods at present available, or how far these can be developed to meet the new problems that arise, only subsequent work can show. Since the completion of the work of which the present paper is an account, considerable progress has been made in a number of directions, and the writer believes that it will be possible by work along these lines to attack two large

$$
1 \text { Ann. Bot., xxxvi, p. } 257,1922 .
$$

[Annals of Botany, Vol. XXXVII, No. CXLV, January, 1923.] 
problems of fungal physiology. One of these is the problem of strainsi. e. accepting the existence of several strains of the same organism, what physiological differences can one establish between them, and can one correlate with these any of the distinct appearances of the particular strains ? Such an investigation is much required in these days, when numerous strains are being discovered in many organisms. If in these cases, as is generally conceded, the morphological criteria are insufficient, and therefore recourse has to be had to physiological means of differentiation, it is obviously important to gain some idea as to what physiological characters will be of value in this connexion, i. e. what physiological characters are sufficiently constant and determinable with sufficient accuracy to be of use from the systematic standpoint.

The second problem may be described as that of the 'suitability' of a particular nutrient for a particular fungus. On this basis one may attempt to interpret the different growth appearances of an organism on one medium as compared with those on another, and in particular to explain the fact of an organism growing well on one medium and not at all on another. This latter problem is of the greatest interest in pathology, as its solution would form a vantage ground for the study of immunity in so far as the latter is based on nutritional factors.

The present paper deals with the so-called 'staling' of fungal cultures. The term 'stale' and its derivatives are in common laboratory use, and as some such term will be required in the present account, if much circumlocution is to be avoided, it is proposed to adopt the term throughout. By a 'stale culture' is understood one which has ceased or practically ceased growing; by a 'stale medium' one understands a medium which, through the growth in it of an organism, has been made useless, or nearly so, for further growth of the same or other organism. By 'staling substances or products' one means those metabolic products of the organism which are responsible for slowing down or stopping its growth. The use of this last term tacitly assumes that the cause of the slowing down of growth in a culture is the presence of products due to the organism and which are deleterious to growth, and that the staling is not to be ascribed merely to the removal from the medium of certain food substances: for many fungi, at any rate, this assumption appears to be fully justified. It is interesting to note that the phrase commonly used in German literature in this connexion is 'gebrauchte Nährlösung', a phrase which refers simply to the history of the cultural solution and not to any properties which it may have developed in consequence of that history.

In defining a particular word for use in this connexion one must take into account the present state of knowledge on the subject. Without going into details, one may state it as follows: The earlier workers, e.g. Duclaux, ${ }^{1}$

1 Duclaux: Traité de Microbiologie, 1900. Ref. in Lafar's Handbuch, vol. i, p. 504, 1907. 


\section{Fungi on Culture Media.}

believed that a 'used solution' is necessarily a stale solution-that is, that the growth in a medium of a particular organism rendered the medium less fit for further growth, at any rate of the same organism. Even to-day this rule will probably be found to cover the great majority of cases. But exceptions have been shown to exist, e.g. in a more recent work of Nikitinsky, ${ }^{1}$ according to which a certain amount of growth of a fungus in a medium may render the latter more suitable for the further growth of the same fungus. A special and very important illustration of the same thing is the so-called 'Bios' effect described by Wildiers. ${ }^{2}$ Here it was found that the growth of an organism in a fresh cultural solution was enormously accelerated by the transference with the inoculum of some of the metabolic products from the older culture, and in some cases this transference was indispensable. Thus it is clear that a ' used solution' is not necessarily a stale solution in the common acceptation of the term stale. It is of course possible that the accelerating substances which must be postulated to explain Nikitinsky's and Wildiers's results are the same as the staling substances, the difference in effect being due simply to difference in concentration. In that case the extension of the term 'staling' to include the effects of stimulation could be defended on grounds of analogy with, for example, toxic substances. Thus it is usual and justifiable to describe copper sulphate as toxic to fungi even though it has been demonstrated that it stimulates fungal growth when present in very minute quantity. However, the identity of the active growth affecting substances in the case of fungi has not been demonstrated, and any extension of the term 'stale' along the lines indi cated above is not at present justified. Further, any definition of staling cannot, in the present state of our knowledge, be based on the 'staling substances' themselves, which are in the main substances of unknown chemical nature, the existence of which is postulated to explain observed effects. The definition must be made on the basis of the observed effects.

The question now remains as to what are the effects observed. These will appear in the sequel, but it may be said here that the general rule is that the rate of spread of a fungal colony increases to a maximum at which it remains steady or from which it subsequently declines. Evidence will be brought forward to show that this decline from the maximum rate of growth can be controlled and modified in a variety of ways, all of which indicate that certain products of the metabolism of the fungus are affecting the medium at the growing margin in such a way as to cause a retardation of growth, and further, that if these products could be removed or otherwise rendered inoperative, the colony would continue growing at the maximum rate. A colony, therefore, which, though kept under the same external conditions, is not growing in diameter at the same rate as formerly, will be

1 Nikitinsky: Jahrb. f. wiss. Bot., 1904, xl. I.

2 Wildiers: Koch's Jahresber., I901, xii. I33. 
described as showing staling at its growing margin, and in accordance with the amount of falling off will be described as more stale or less stale as the case may be. Further, as the basis of measurement is that of increase in diameter, the term staling will in the present paper have reference to growth at the margin of the colony only.

\section{Experimental Method.}

The general method of experiment was the same as that described in the earlier paper dealing with the growth of fungal colonies (loc. cit., p. 270), so that it is unnecessary to repeat the details here. Certain further particulars will be given below in describing particular experiments.

The fungi mainly dealt with were Sphaeropsis malorum and a species of Fusarium, ${ }^{1}$ both of which show well-defined staling phenomena. Both have also advantages in this respect, that there is no trouble in either case from air-borne spores, the former only producing spores, if at all, in very old cultures, the latter forming its spores in moist masses. Fungi which possess air-borne spores are troublesome in this kind of work, as the disturbance incidental to measurement is liable to scatter spores on to the uninvaded portion of the plate, thereby producing colonies which interfere with the free growth of the mother colony. The removal of the lids of the Petri dishes for purposes of measurement brings in the risk of contamination from outside, and this has to be guarded against. This trouble has been met in two ways-by disinfecting the laboratory at fairly frequent intervals and by cutting out contaminating organisms when they are first noticed. By carrying out these precautions and by avoiding any work with such fungi as Penicillium glancum and Rhizopus nigricans during the course of these experiments, no appreciable trouble was met with in the way of contamination of the plates from outside.

The stock cultures from which the inocula are taken are of course kept pure in the usual way in sterile tubes.

The actual measurements were made in each case in two directions at right angles to each other, each measurement being made to the nearest half-millimetre. In cases where the colony for some reason or other had not grown circularly, measurements were made along the long and the short diameter, and the average taken. When the greatest accuracy was required, successive measurements were made along marked directions.

When the object was to compare successive daily growths with each other, the cultures were kept at uniform temperature in an incubator. In a large number of experiments, however, where it was only sought to draw comparisons between different cultures under the same temperature condi-

1 This is the same fungus as was dealt with in the earlier paper already cited. Its systematic position will be set out in a subsequent publication. 
tions, the cultures were grown side by side in diffuse light at the general laboratory temperature.

The method of studying growth by increase of diameter offers certain contrasts with that of dry-weight measurements. A comparison of the two methods may conveniently be given here.

As regards simplicity the former has obviously great advantages. Thus it is possible to carry out experiments on a large scale, a circumstance which allows of abundant repetition of experiment and effective control in each case. There is, further, the very great advantage that the growth of any one colony can be followed throughout all its stages, whereas the dryweight method involves the destruction of a culture for each measurement taken.

A second point of comparison is that the method of linear measurement is suited to growths on solid media (agar, gelatine, \&c.), while the method of weight measurement can only be carried out effectively in the case of cultures in liquid media. It is certainly possible to determine the dry weight of a culture on gelatine media by dissolving away the latter with gentle heat, a process which can be carried out without killing the fungal mycelium, and thus without appreciable loss of cell contents. A similar method is not available for the case of agar media, and if it could be shown that reliable figures were obtainable by taking the dry weight of the killed (and presumably extracted) fungal mycelium after removal of the agar by high temperature, the fact remains that that method would be extremely laborious. This circumstance is unfortunate, as agar is in general a much more suitable solid medium than gelatine, partly by reason of convenience, but especially on account of the fact that gelatine is broken down by many fungi, and for that reason cannot be used in experiments where relations between growth and nutrition are being tested. Thus the method of dry-weight measurement, though well suited to the study of, for example, yeasts and such fungi as normally live in a liquid substrate, is not well adapted to the conditions under which fungi are generally grown and studied in the laboratory, viz. on solid media. Moreover, it should be noted that the method of studying the growth in terms of the spread of a colony from its point of inoculation is more calculated to throw light on some important problems, such as the spread of a parasitic organism from its point of infection over the host tissue, than is likely from studies by the dry-weight method. In the latter case the conditions are essentially different, involving as they do the simultaneous development of a large number of colonies in the medium with mutual interference from the start.

The greatest objection to the method of linear measurement is that it affords in many cases no indication whatever of the amount of mycelium in the fungal colony. Thus two colonies of the same diameter may possess vastly different amounts of mycelium, a fact which may be readily 
visible to the eye though an exact measurement of the difference may be difficult to obtain. In fact in many cases there is a direct correlation between vigorous marginal growth and scantiness of mycelium within the culture.

But while the method of linear measurement gives in general no indication of the amount of growth made, it is well to remember that the alternative method is not free from objections in this respect. Numerous contradictions have arisen in the literature of fungal nutrition from misinterpretation of the results of dry-weight experiments. An example will suffice to show how errors have arisen. Suppose the object is to compare the nutritive value of two substances or two cultural media, A and B, for a particular fungus. The simple method is to start the fungus on the two media and at the end of an arbitrary time, say a fortnight, determine the dry weights of the mycelia produced in each case. Working in this way different investigators have come to the most contradictory results. The source of the apparent contradiction lies in the fact that the same fungus may have a growth-time relationship widely different on different media. In one case the increment of growth may be rapid at first and then reach a moderately low limiting value: in the other it may slowly increase to a high maximum. Again, the curve of dry weight of a fungus does not remain at a steady value but again diminishes through degenerative changes. Thus the dryweight method cannot be safely used unless one is prepared to follow the growth at short intervals throughout its whole history. Short of that, the results will have no value. These considerations greatly increase the labori, ousness of the dry-weight method.

All things considered, one may safely say that the method of linear measurement is the best to apply in exploratory work. By this means one can indicate problems of limited dimensions to which the more searching method of dry-weight determination can profitably be applied.

There remains the question of the relative accuracy of the two methods. The advantages, quite apart from the number of controls that can be employed, appear to rest with the method of linear measurement. Experience in this laboratory has shown that it is difficult to obtain an even curve of growth as determined by dry-weight measurement, as irregularities tend to appear under apparently identical conditions among the various cultures. In linear measurements of growth, the degree of uniformity obtainable depends on a variety of factors. With the same fungus, it may vary with the medium ; thus it is difficult on some media to get uniform starting of the different controls while it is quite easy on others. Again, the degree of uniformity varies with the phase of growth of the colony, and it will be shown subsequently that the tendency to lack of uniformity among the various controls is an indication that staling is taking place.

As regards the two fungi mainly dealt with in this paper, Sphaeropsis 
and Fusarium, the experience of the writer is as follows: Uniformity of starting is somewhat greater with the latter ; thus it is possible to place very similar masses of Fusarium spores on the plates, and, within wide limits of size of inaculum, the initial growth of the resultant colony is the same in all cases. With Sphaeropsis, the inocula are small portions of mycelium and it is not so easy to ensure uniformity of inoculation. The resultant young colonies vary more among themselves than is the case with Fusarium, but it has been found that if the first two days' growth be subtracted from the following readings, much better agreement is shown. Under suitable conditions the degree of uniformity observed is surprising, and it is a common result to find the various controls agreeing among each other to within a few millimetres over a diameter of Io centimetres.

The following graphs (Figs. I-6) will serve to illustrate the different types of growth met with in different fungi, and with the same fungus under different conditions, and they will indicate the nature of the problems which arise in a study of this description.

In the experiment from which Figs. I-3 were constructed, the various fungi were grown in the usual way on somewhat deep ( $\mathrm{I} \mathrm{cm}$.) layers of potato agar in closed Petri dishes. The medium was the same in all cases, and the growth tests were run concurrently. The experiment was done in triplicate, and each measurement is the average six readings, i. e. of two from each culture in the set. The ordinate corresponding to each plotted point on the curves represents the increment in diameter in the preceding twenty-four hours.

An examination of the curves in Fig. I brings out the following particulars :

In the early stages, the growth-rate increases day by day, so that the curve of total growth is convex to the time-axis.

In the $15^{\circ} \mathrm{C}$. curve, the growth-rate had not reached a steady value at the end of the experiment, by which time the culture had reached a total diameter of $10 \mathrm{~cm}$. and had almost covered the whole surface of the medium. The $20^{\circ}$ curve, on the other hand, showed signs of reaching a steady growthrate: and in the curve of growth at $25^{\circ}$ this limiting value had been reached, and a subsequent slight falling off recorded. This falling off is very pronounced in the $30^{\circ}$ curve, where growth ceased entirely after the first few days.

The general statement of these results is that the growth-rate increases to a maximum which is sooner reached the higher the temperature. At the two higher temperatures a decline in growth-rate sets in, which is more pronounced the higher the temperature. Whether such a falling off would appear at the two lower temperatures if the cultures were grown on a larger surface of medium was not determined. It was sufficient for the present purpose to show that this fungus would grow to a diameter of $10 \mathrm{~cm}$. at 
ordinary temperatures without showing any signs of marginal staling. Staling effects, however, do appear at $25^{\circ}$, and especially at $30^{\circ}$, but it will be noted that both these are supra-optimal temperatures. Whether these effects were due to actual staling substances appearing in the medium, or to direct injury to the fungal hyphae from prolonged exposure to the high temperature, was not investigated. ${ }^{1}$

Fig. 2 gives the corresponding results for Alternaria grossulariae.

The curve for growth at $30^{\circ}$ is not drawn in, as it is practically identical with that at $25^{\circ}$. This fungus shows somewhat different growth features from those of Botrytis. It will be noticed that we have here a more slowly growing fungus with higher cardinal points for temperature. A striking

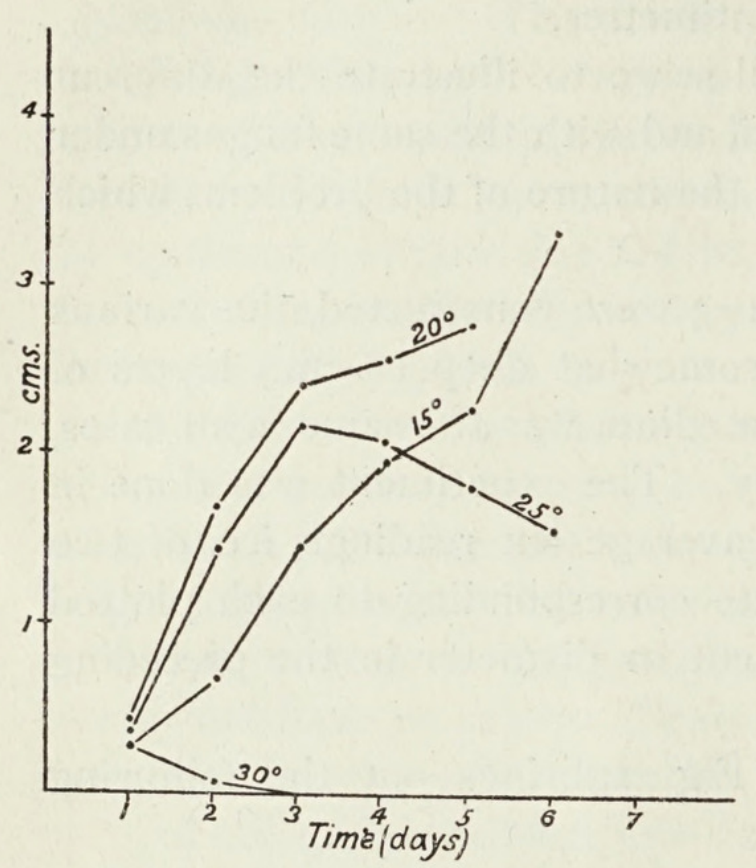

FIG. I. Botrytis cinerea on potato agar.

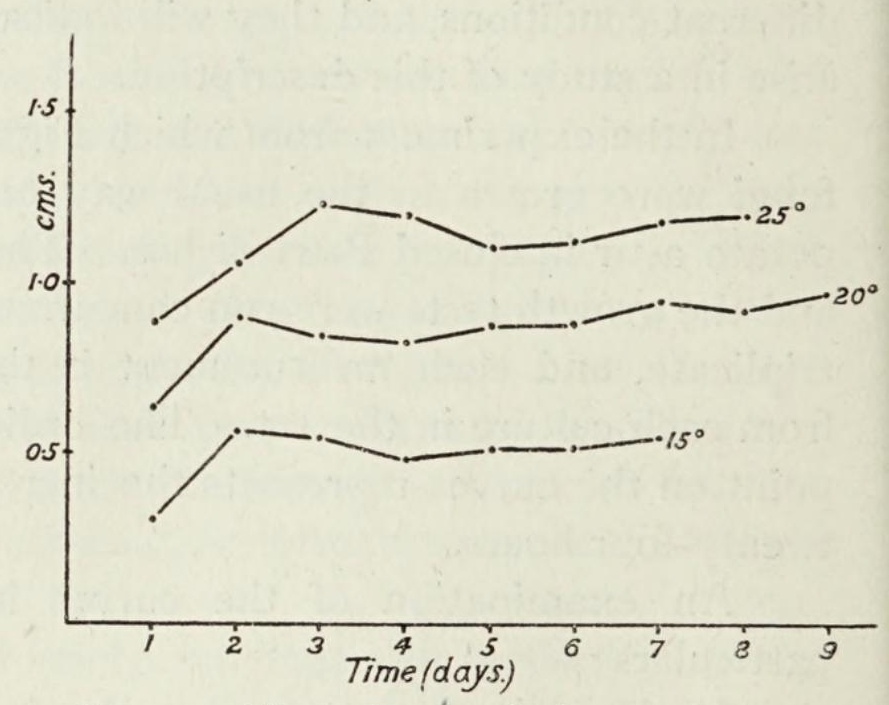

FIG. 2. Altemaria grossulariae on potato agar.

difference also is the shortening of the initial period of accelerated growth, so that all the cultures have reached their limiting growth-rate by about the second day. From that time onwards, all the curves are roughly parallel to the time-axis, so that the growth-rate in each case on the eighth day is not sensibly different from that on the second. There is no indication of staling in any case.

Both the preceding fungi may be cited as illustrations of the nonstaling type, i.e. putting aside the case of the $30^{\circ}$ curve for Botrytis cinerea which brings in considerations of supra-optimal temperature and is outside the scope of the present paper.

Fig. 3 illustrates the staling type of growth, with other features similar to those described in Fig. I and which need not be repeated here. The essential point to notice is that, in contrast with the first two cases, there is distinct staling of the fungal colonies at all the temperatures. In

\footnotetext{
1 Compare, on this point, Balls, Ann. Bot., I 908, xxii. 557.
} 
all cases the rate of growth at the seventh day is much reduced from what it was on the second and third days.

One can obtain all gradations from those fungi which would, as far as can be séen, grow on indefinitely at the same rate to those which form small colonies and then cease growing entirely. Fungi of the latter type are sometimes referred to as showing 'limited growth', but there are little grounds for thinking that there is anything absolute in their limitation. They are simply to be looked upon as fungi which have vẹry strong 'staling tendencies', and one could anticipate that, if means were devised for removing or reducing in intensity the staling products, the amount of growth would cease to be limited.

A simple and effective way of demonstrating that the slowing down of the marginal rate of growth is not due to anything inherent in the growing



FIG. 3 Fusarium sp. on potato agar.

hyphae, but is caused by staling products diffusing outwards from the mycelial mass of the colony, is to cut away day by day the central portion of the colony, leaving only a fine fringe of marginal mycelium. Experiments of this type were carried out with the present and with another more strongly staling Fusarium. It was seen that the difference due to cutting out the central region of the colony was imperceptible when the fungi were grown on a medium on which no marginal staling took place (e.g. a dilute potato extract with agar), whereas when the medium was one which gave staling (e.g. potato agar of standard strength), the effect of cutting out the central portion of the colony was to postpone the incidence of staling. An illustration of the latter result is given in Table I.

\section{TABLE I.}

\begin{tabular}{|c|c|c|c|c|c|c|c|}
\hline \multirow[t]{2}{*}{$x^{2}$} & \multicolumn{7}{|c|}{ Growth during } \\
\hline & $\begin{array}{l}\text { First } \\
2 \text { days. }\end{array}$ & $\begin{array}{l}3 r d \\
\text { day. }\end{array}$ & $\begin{array}{l}4^{t h} \\
d a y .\end{array}$ & $\begin{array}{l}5^{t h} \\
\text { day. }\end{array}$ & $\begin{array}{l}\text { 6th } \\
\text { day. }\end{array}$ & $\begin{array}{l}\text { 7th } \\
\text { day. }\end{array}$ & $\begin{array}{l}8 t h \\
d a y .\end{array}$ \\
\hline Uncut & 0.85 & 0.85 & 0.9 & 0.7 & 0.5 & 0.3 & 0.3 \\
\hline Cut & 0.85 & 0.85 & 0.9 & 0.78 & $0.6_{5}$ & 0.63 & 0.68 \\
\hline
\end{tabular}


The amount of staling shown by a particular organism varies from one medium to another and is dependent upon the amount of the particular medium present. Figs. 4 and 5 illustrate this point.

Fig. 4 gives the daily growth-rates of Sphaeropsis on apple extract agar (A.A.), and potato extract agar (P.A.). In each case the medium is presented in large or small quantity by varying the depth of pouring. In one set the plates are deep (about I cm.) and in the other shallow (about $2 \mathrm{~mm}$.). The ordinate corresponding to each plotted point represents the average daily growth since the preceding reading. The points brought out by this graph are: (I) that there is distinct staling on potato agar, but none on apple agar; (2) that the rate of spread of the cultures on shallow potato agar is much less than on the deep medium, whereas both shallow and deep platings grow at approximately the same rate on apple agar.

A large number of experiments have been performed on the same lines

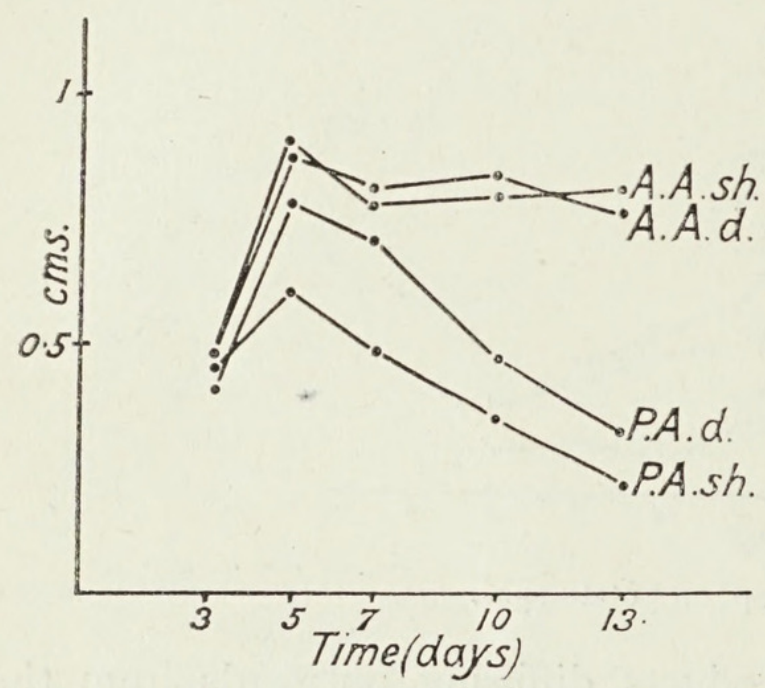

FIG, 4. Sphaeropsis malorum on apple agar (deep and shallow) and potato agar (deep and shallow).

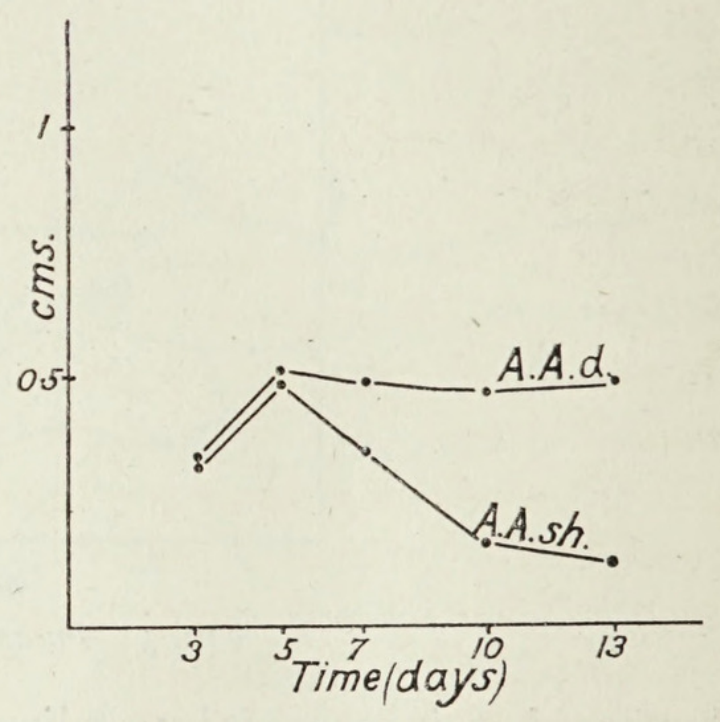

FIG. 5. Fusarium sp. on apple agar (deep and shallow).

as those illustrated in Fig. 4, and it is possible to generalize. When the depth of medium is varied, it is found that the rate of growth on the deep and shallow plates is at first identical. If the fungus does not stale on that particular medium, the rates of growth on the deep and shallow plates continue the same. If the fungus stales, then the rate of growth on the shallow plates after some time is different from that on the deep plates. In the present case the growth on the shallow plates lags behind the deep ones, and this is the rule on most ordinary media. But there are interesting exceptions to this rule which will be described in a subsequent paper.

This method of comparing the growth on deep medium with that on shallow has proved to be of great value, as one can obtain a good idea from inspection of the two sets of cultures as to how far staling is taking place.

Fig. 5 gives the growth of Fusarium on apple agar. In this case there is obvious staling on the shallow plates, though it had not appeared in a pronounced form in the deep plates. This experiment illustrates the 
fact that Fusarium stales under conditions in which Sphaeropsis does not; this is in accordance with a large body of evidence which indicates that Fusarinim is a fungus with stronger staling tendencies than Sphaeropsis.

The amount of staling shown can also in certain cases be modified by a slight alteration of the conditions under which the experiment is set up. Fig. 6 is an example of this. Both graphs represent the growth of Sphaeropsis on shallow layers of potato agar, the difference being that in the one case (the lower graph) growth took place in closed Petri dishes, in the other the Petri dishes were placed with the lids off in large five-litre containers. The difference was simply one of the volume of atmosphere in direct communication with the growing fungus, and it was sufficient to influence distinctly the incidence of staling. This type of experiment has

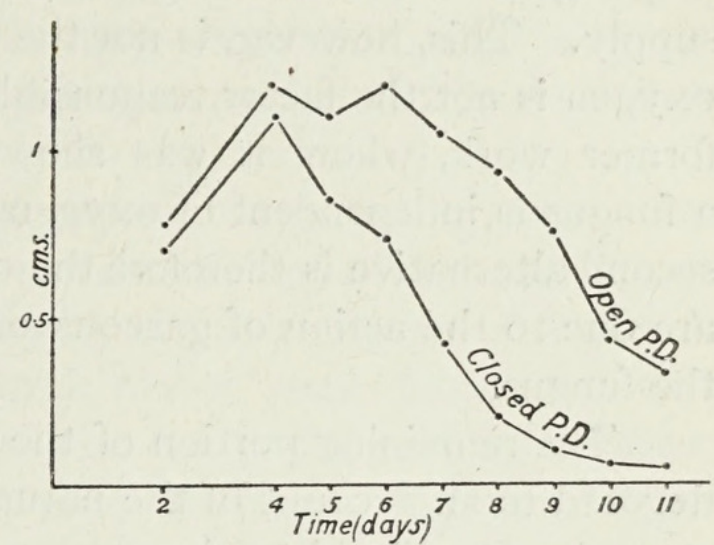

FIG. 6. Sphaeropsis malorum on potato agar. been repeated many times under varying conditions with Sphaeropsis on potato agar culture, and the above result has been conficmed in every case. In the case of Fusarium, the same result is obtained, but as a rule to a less marked degree.

The foregoing result indicates that we are dealing here with an effect produced by differences in the conditions of gaseous exchange in the two cases. The two main possibilities which suggest themselves are (I) that in the closed Petri dishes limitation of oxygen supply is the cause, (2) that a gas which retards growth tends to accumulate in the closed Petri dishes.

Table II, which is a fuller record of the experiments from which Fig. 6 was taken, indicates which of the above alternatives is the likely one. The figures in the table represent the total diameter (in $\mathrm{cm}$.).

TABLE II.

Total growth of Sphaeropsis on Potato agar, T. I4-I $5^{\circ}$ :

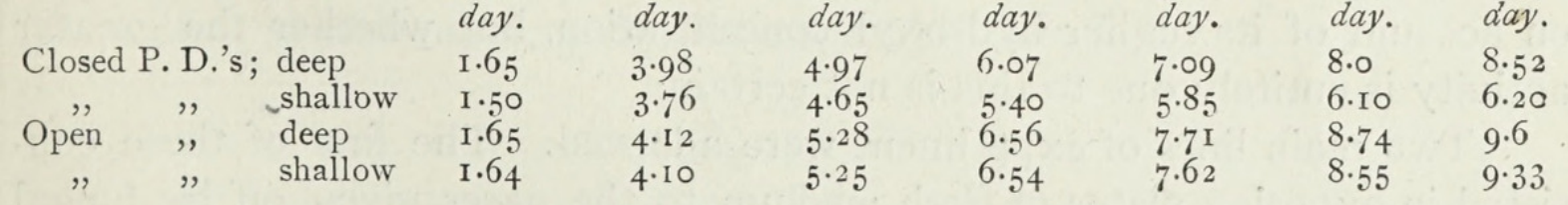

From this table it is seen that the effect of opening the Petri dishes is to increase the growth both on the deep and shallow plates, but especially on the latter. The shallow closed plates show very strong staling, the corresponding deep plates show definite staling at about the eighth day, while the cultures in the open plates only show slight staling at the end of the experiment, so little in fact that the deep and shallow plates are still 
growing at almost the same rate. Now the Petri dishes are of approximately the same volume, and if limitation of oxygen is the factor concerned in staling, one would anticipate that staling would appear more strongly in the closed Petri dishes with deep layer of medium than in the corresponding ones with shallow layer of medium, first because there is greater free air-space in the latter, and secondly because there is throughout greater fungal growth in the former and consequently greater demands on oxygen supply. This, however, is not the case, whence it follows that the supply of oxygen is not the factor responsible. This conclusion is in agreement with former work, where it was shown that the amount of growth made by a fungus is independent of oxygen pressure within very wide limits. ${ }^{1}$ The second alternative is therefore the one indicated, i.e. the differences observed are due to the action of gaseous or volatile products of the metabolism of the fungus.

The remaining portion of the experimental part of this paper 'will be devoted to an account of the nature of this action and how it can be demonstrated. It will aid to clearness to state here the main conclusions arrived at. The two gaseous products concerned in this differential action are carbon dioxide and ammonia. The former arises of course in the ordinary process

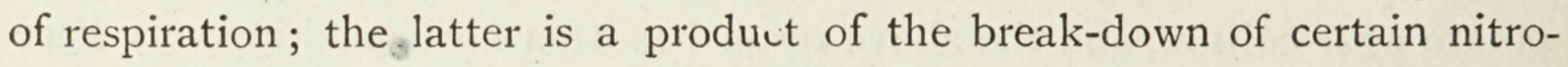
genous compounds (proteins, peptones, amides) which are present in most media, e.g. in potato and in all media containing gelatine. According to the extent to which these products accumulate or are disposed of, the amount of staling is affected. The ammonia, which is produced on some media in an amount greater than the fungus can take up, dissolves in part in the medium and renders it stale, or, more correctly, makes it more stale than it would be if this product were removed. The staling action of ammonia is partly counteracted by the carbon dioxide, and in accordance with the experimental conditions which allow the one or the other to accumulate there will be greater or less staling. From the physico-chemical point of view the principles involved are better stated as follows: When carbon dioxide is in excess, the ammonia goes into solution in the medium in the form of the bicarbonate; when ammonia is in excess, as ammonium carbonate and free ammonia. The latter is the more actively staling combination, probably on account of its higher hydroxyl concentration, but whether the greater activity is entirely due to this is not certain.

Two main lines of experiment were adopted. The first of these consisted in exposing plates of fresh medium to the gases given off by fungal cultures, after which the staling action of the exposed plates was tested by inoculating them with various fungi. The second method consisted in growing fungal colonies in atmospheres which were controlled in respect of their carbon dioxide and ammonia content.

The method of experiment in the first case is very simple. A batch of 1 Loc. cit., p. 266. 
plates, as uniform as possible (i. e. containing the same depth of the same medium), was inoculated with the fungus to be tested. When these cultures had reached a certain age a second batch of plates of the same size was prepared, and one of the second batch, still without inoculation, inverted over each culture of the first batch, the lids being removed. Similar uninoculated plates were kept unexposed as controls. After one to two days' exposure all the plates of the second batch were removed and their lids restored as rapidly as possible, after which they were inoculated with the same or another fungus, and the growth in a given time, usually two days, determined.

As an illustration the following experiment may be quoted. The staling was produced by ten-day-old cultures of Sphaeropsis on potato agar. The exposed plates had shallow layers of potato agar. After three days' exposure, the exposed and control plates were inoculated with Sphaeropsis. The diameter of the new growths in two days were as follows:

On unexposed plates (eleven colonies) . $\mathrm{I} \cdot 57 \mathrm{~cm}$. (max. I.65, min. I.5).

On exposed plates (twelve colonies) . . $0.42 \mathrm{~cm}$. (max. 0.5 , min. 0.3 ).

This staling effect soon disappears if the exposed plants are allowed to remain for some time with their lids off before inoculation. Also, the growth on the exposed plates tends to recover its normal rate as time goes on, probably by gradual escape of the staling gas. Again, the staling effect is more pronounced if the exposure is continued after inoculation.

A large proportion of these experiments were carried out before the nature of the staling substance was recognized. Cultures on potato agar, where the evolution of ammonia is small and not readily recognizable as such by its smell, were employed, and so a large number of control experiments were made to eliminate other factors. Two of these may be mentioned. A certain amount of drying of the exposed plates takes place during the process, but tests showed that this was of no importance. Also the carbon dioxide evolved by the cultures plays no part. Plates exposed in 40 per cent. carbon dioxide show no signs of being stale after removal from the gas. The absorbed carbon dioxide is apparently dissipated with great rapidity.

Deep plates of a given medium are more slowly staled by this treatment than shallow ones, as is illustrated by Table III.

\section{TABLE III.}

Plates of Potato Agar exposed to Cultures of Sphaeropsis on Potato Agar.

\begin{tabular}{|c|c|c|}
\hline & \multicolumn{2}{|c|}{ Growth of Sphaeropsis in } \\
\hline Unexposed, deep & $\begin{array}{c}\text { First two days. } \\
\mathbf{I} \cdot 9 \mathbf{I}\end{array}$ & $\begin{array}{c}\text { Second two days. } \\
2.54\end{array}$ \\
\hline ,, shallow & $\mathrm{I} \cdot 94$ & $2 \cdot 37$ \\
\hline Exposed (two days), deep & $\mathrm{I} \cdot 77$ & $2 \cdot 35$ \\
\hline shallow & $\mathrm{I} \cdot \mathrm{I} O$ & 2.07 \\
\hline
\end{tabular}

These figures also illustrate recovery from staling. 
With the same depth of pouring, some media are more readily staled than others, e. g. potato agar is more readily staled than potato gelatine (with the same concentration of potato extract in each), as is shown by the following figures, which represent two days' growth of Sphaeropsis:

\section{TABLE IV.}

\begin{tabular}{|c|c|c|}
\hline & Unexposed. & $\begin{array}{l}\text { Exposed to } \\
\text { Sphaeropsis } \\
\text { Culture on } \\
\text { Potato Agar. }\end{array}$ \\
\hline Potato agar (agar I. $5 \%)$ & 2.08 & $\mathrm{I} \cdot 03$ \\
\hline Potato gelatine (gelatine $10 \%$ ) & I. 84 & I. 83 \\
\hline
\end{tabular}

Any of the usual media (plum, prune, apple, \&c.) made up with gelatine behave in the manner indicated.

Under the same conditions some fungi are more easily staled than others. The following table (V) illustrates the behaviour of four fungi in this respect. The cultures used to produce the staling were Sphaeropsis on potato agar and potato gelatine, both series being of the same age. The exposed plates were of potato agar. The figures in round brackets represent the percentage growth, that on the unexposed plates being taken as 100.

TABLE V.

\begin{tabular}{|c|c|c|c|}
\hline Fringus tested. & Unexposed. & $\begin{array}{l}\text { Exposed to } \\
\text { Sphaeropsis } \\
\text { on P.A. }\end{array}$ & $\begin{array}{l}\text { Exposed to } \\
\text { Sphaeropsis } \\
\text { on P.G. }\end{array}$ \\
\hline Botrytis cinerea & $\mathrm{I} \cdot 82(\mathrm{I} 00)$ & $0.64(35)$ & 0.0 \\
\hline Sphaeropsis malorum & $\mathrm{I} .88(\mathrm{r} 00)$ & $0.83(44)$ & \\
\hline Fusarium sp. & $1 \cdot 5^{2}(100)$ & $1.18(77)$ & $0.74\left(4^{8}\right)$ \\
\hline Penicillium glaucum & $0.94(100)$ & $0.79(84)$ & $0.45(48)$ \\
\hline
\end{tabular}

A review of the experiments made on this point shows that Botrytis cinerea is more readily staled than Sphaeropsis, which again is more sensitive than either Fusarium or Penicillium. The two last are not definitely distinguishable in this respect.

As to the nature of cultures showing staling, the magnitude of the effect depends on age. For the first few days it is negligible, then it reaches a maximum, and in old cultures it again becomes small. The period of most active evolution of ammonia was not accurately determined, but the effect is usually well seen in cultures of ten days to a month. The effect appears sooner in shallow than in deep cultures, though after a time the latter show it just as strongly.

The effect also depends on the fungus used. With potato agar as medium, Fusarium and Sphaeropsis produce staling in a marked degree, Botrytis only very slightly, and Monilia fructigena not at all.

The composition of the medium on which the fungus is grown is of great importance. There is no evolution of staling gas from cultures on Richards' solution with agar. 
It is interesting to compare the growth curve of Fusarium or Sphaeropsis on potato agar with that on potato gelatine (same potato extract in each case) in the light of the results shown in Table V. The difference between the growth curves on potato agar and potato gelatine consists in this - that, other conditions being the same, staling is shown sooner on potato agar, but subsequently it becomes more intense on ${ }_{4}$ the gelatine medium. This result is explicable on the grounds of two factors: (I) the greater evolution of ammonia from the gelatine cultures as growth proceeds ; (2) the greater capacity of gelatine media to absorb free ammonia without becoming alkaline. The first of these factors is illustrated in the table just given; the second has already been illustrated in the experiments dealing with the staling of fresh media when exposed to fungal cultures (Table IV). A more instructive illustration is given in Table VI. To equal volumes of potato agar and gelatine equal quantities of a series of dilutions of ammonia were added, and the $\mathrm{P}_{\mathrm{H}}$ of each determined colorimetrically. The concentrations of ammonia added were in the order 25,5 , I.

\section{TABLE VI.}

\begin{tabular}{|c|c|c|c|}
\hline & Medium. & $\begin{array}{c}\text { Ammonia } \\
\text { added. }\end{array}$ & $P_{H} \cdot$ \\
\hline Potat & elatine ( & 0.0 & $5 \cdot 4$ \\
\hline ", & ", $\quad "$ & 1.0 & $5 \cdot 4$ \\
\hline$"$ & ," & $5 \cdot 0$ & $6 \cdot 3$ \\
\hline Potat & gar (I. $=\%$ agar) & $\begin{array}{r}25 \cdot 0 \\
0.0\end{array}$ & $\begin{array}{l}9 \cdot 2 \\
6 \cdot 4\end{array}$ \\
\hline , & " & 1.0 & $8 \cdot I-8 \cdot 2$ \\
\hline ", & ," & $5 \cdot 0$ & $9 \cdot 5$ \\
\hline ," & ", & $25 \cdot 0$ & too high to me \\
\hline
\end{tabular}

This table shows that the addition of a small amount of ammonia to potato agar has a comparatively greater influence in diminishing its $\mathrm{H}$-ion concentration than is the case with potato gelatine. The earlier incidence of staling in the cultures on potato agar is readily understandable in the light of this result.

Incidentally one may note that agar media are preferable to gelatine media for the purpose of keeping stock cultures, at least in the case of certain organisms. The large evolution of ammonia that may occur on gelatine media may be injurious or even fatal to the organism. ${ }^{\mathbf{1}}$

The evolution of an alkaline gas can be shown simply by placing a piece of litmus paper in the lid of the culture. Extended observation has shown that the more rapidly a particular culture changes the colour of red litmus paper placed in the lid, the more vigorously does it produce staling in a plate of fresh medium exposed to it, and in particular where no alkaline gas can be demonstrated by means of litmus, no staling effect on a plate

1 For an illustration of an effect of this nature see Boas, Ber. d. deut. bot. Gesell., xxxvii, p. 63 , 1919 . 
of medium is produced. Further, a plate of medium on exposure to a culture of Sphaeropsis is protected from staling, at any rate in the neighbourhood of the watch-glass, by the insertion between the two of a small watch-glass containing dilute acid.

That the gas was ammonia was shown by its giving Nessler's reaction. It was also jdentified by isolation as the chloride. Alkylamines might be present in small quantities, but they were not demonstrated.

In order to be certain that the cultures producing the staling were absolutely free from any bacterial contamination (which cannot be guaranteed in Petri dish cultures where the lids have been removed), the experiments were carried out with pure tube-cultures of the staling fungus. The medium to be staled was used in the form of a film on sterilized slides. The results obtained were identical with those already described.

The amount of ammonia present in tube cultures was determined for a number of media. The tubes contained 25 c.c. of the medium, half of them being inoculated with Sphaeropsis, the other half left uninoculated. After four weeks' growth (at $18^{\circ} \mathrm{C}$.) of the inoculated tubes, the contents of each tube were distilled over magnesia, and the distillate collected in decinormal sulphuric acid. The amount of alkaline gas passing over was then determined by titrating against decinormal alkali. The figures in the table represent cubic centimetres of decinormal solution. For each medium, eight tubes were analysed, i. e. four inoculated and four uninoculated. The results, which showed good agreement among themselves, gave the average values shown in the following table:

\section{TABLE VII.}

\section{Agar $1.5 \%$; gelatine $10 \%$.}

Medium.

Potato agar

Potato gelatine

Richards' solution $\left(\mathrm{KNO}_{3} \mathrm{I} \%\right)$ with agar

$\left(\mathrm{KNO}_{3} 1 \%\right.$ ) with gelatine

$\left(\mathrm{KNO}_{3} 0.1 \%\right.$ ) with agar

$\left(\mathrm{KNO}_{3} \circ .1 \%\right)$ with gelatine
Amount of Alkaline Distillate from Inoculated Tubes. Uninoculated Tubes.

$\begin{array}{cl}3.03 & 0.98 \\ \mathrm{I} \cdot 7 & 2.03 \\ 0.5 & 0.17 \\ 9.3 & 0.3 \\ 0.28 & 0.13 \\ 13.55 & 0.6\end{array}$

We see here that there is a moderate amount of ammonia present in the cultures on potato agar, and much more on all the cultures of gelatine media (including, from other experiments, apple gelatine). The small quantities obtained with the two Richards' solutions plus agar fall within the experimental error limits of the determinations. Though the amount of ammonia present in these gelatine media is considerable, it is not all present in the free state, being neutralized up to a certain limit by the acidity of the nutrient solution and of the gelatine itself. With media such as apple gelatine and Richards' solution gelatine, where the nutrient solution is itself fairly acid, free ammonia is late in appearing in the 
culture. As potato extract is practically neutral, free ammonia appears sooner in cultures on potato gelatine.

It now remains to show how far the ammonia given off by a fungus is responsible for slowing down the growth of the fungus itself, and also to demonstrate what part the carbon dioxide of respiration plays in the staling process.

The general method of experiment was to compare the growth of a fungus on a given medium in closed Petri dishes with the simultaneous growth of the same fungus on the same medium in open Petri dishes. The latter were stacked in 5-litre glass containers with an air-space between each. In the bottom of the containers roo c.c. of a liquid were placed, either water or I per cent. sulphuric acid or I per cent. caustic soda. The initial atmosphere was either ordinary air or air with its carbon dioxide content made up to 5 per cent. It was not possible to obtain incubator space for all the containers used, so all the experiments were carried out at laboratory temperature. As only the relative degree of staling in the cultures as differently set up was of importance, and as each one had the same temperature conditions, the experiments lost nothing in value on this account.

Fig. 6 illustrates the fact that the growth of Sphaeropsis on potato agar is much improved by removing the lids of the Petri dishes and placing the latter in large containers; in addition Io० c.c. water were placed in the bottom of each container. This experiment has been repeated a large number of times and always with a distinct, positive result. The following series of figures is typical of the results obtained, and shows the magnitude of the effect produced. In all the experiments recorded in the table the medium was potato agar, poured deep, with the exception of the ones marked shallow. These experiments were performed at all seasons of the year, and so laboratory temperature varied considerably from one to the other. There is thus no correspondence between the average growth per day in each experiment. Each figure represents the average of four cultures.

\section{TABLE VIII.}

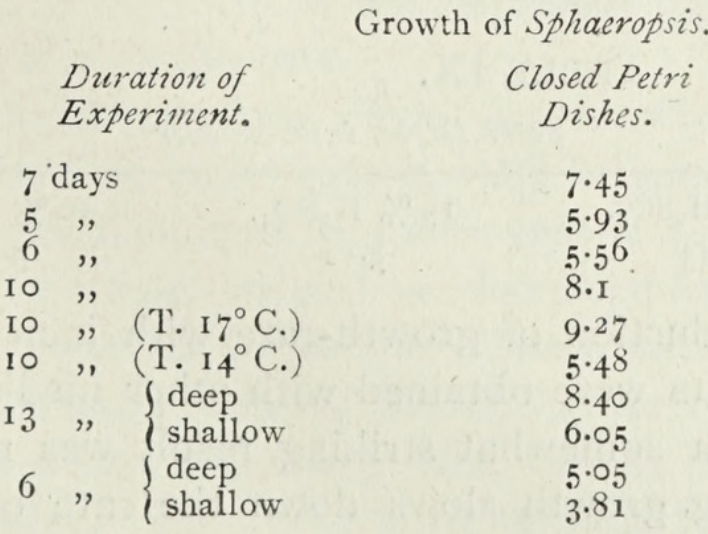

Open P.d.'s in Container over 100 c.c. Water.

8.62

6.04

5.8

$8 \cdot 7$

10.67

$6 \cdot 45$

I0. 50

$9 \cdot 45$

$5 \cdot 38$

$4 \cdot 40$ 
All cultures were examined every two or three days, and at each time of measurement each culture was started anew in ordinary air. By following the growth at intervals it was seen that in the early stages all the cultures were growing at the same rate, but by and by staling set in in the closed dishes, whereas it was later in appearing in the open ones. Cultures of Sphaeropsis on deep layers of potato agar do not stale appreciably for several days. Thus differences of the type recorded above are not striking in the shorter experiments on deep medium. With shallow layers of medium staling sets in sooner, and thus it is found that more striking differences are obtained with shallow plates, as the above table shows.

In accordance with the results here recorded it is possible to take a culture (especially of Sphaeropsis) which has been kept in a closed Petri dish until it shows obvious marginal staling, and then, by removing the lid and placing in a large container in the usual way, to effect a very marked recovery of the rate of growth at the margin. The effect is still more marked if the plates are transferred to an atmosphere containing a moderate concentration ( 5 or to per cent.) of carbon dioxide.

Attempts were made to obtain results similar to those recorded in Table VIII by comparing the growth in closed Petri dishes with that in dishes which simply had the lids raised so that free air exchange could take place, the use of containers being dispensed with. The majority of these experiments failed through contamination at the edges, but even where trustworthy readings could be made the same result was not obtained. The result was unexpected, and an explanation of the apparent discrepancy was sought for in the fact that the cultures thus exposed to laboratory air show a considerable amount of drying up of the medium. Control experiments in which the cultures were inverted over desiccating agents showed that in fact exposure to drying of a plate on which a fungus was growing resulted in slowing down the rate of growth. Plates of various media which had been inoculated with Sphaeropsis were inverted over Petri dishes of the same size containing 15 c.c. of various concentrations of sulphuric acid. The following table shows a typical set of results. The figures represent the growth in four days of Sphaeropsis on potato agar.

\section{TABLE IX.}

Closed Petri Dishes.

Petri Dishes inverted over

$\overbrace{25 \% \mathrm{H}_{2} \mathrm{SO}_{4} \text {. }{ }_{4.55} \mathrm{I}_{5 . \mathrm{I} 2}^{\mathrm{H}_{2} \mathrm{SO}_{4} .}}^{\text {I0 } \% \mathrm{H}_{2} \mathrm{SO}_{4} .}$

There was a gradual reduction of growth-rate with increasing desiccation. Exactly similar results were obtained with other media, e.g. plum gelatine. In this connexion a somewhat striking result was met with, in that while desiccation during growth slows down the rate of growth, a 
comparable amount of drying of the medium before inoculation has no such effect. Obviously this result can only apply within certain limits. Though this line of experiment was not followed out farther, the suggestion seemed to be either that some transpiration effect was coming into play or desiccation of the growing cultures led to a concentration of the staling substances, especially, perhaps, on the surface-layer of the medium.

It was obvious from these control experiments that it was necessary, in using various solutions as gas absorbents, to have the solutions weak in order to avoid any effects due to drying of the cultures. Hence I per cent. sulphuric acid and I per cent. caustic soda were used.

The dependence of staling upon carbon dioxide and ammonia was best brought out by experiments such as those recorded in Table X.

\section{TABLE $X$.}

(a) Growth of Sphaeropsis on potato agar in open Petri dishes in 5-litre containers:

Control.

(In closed P.d.'s.)

8. I

IOO c.c.
$\mathrm{H}_{2} \mathrm{O}$ (air).
8.7

Growth in open P.d.'s in container over
IOO c.c. $\mathrm{H}_{2} \mathrm{O}$

$\left(5 \% \mathrm{CO}_{2}\right)$.

$9 \cdot 4$

(b) Growth of Sphaeropsis on potato agar in closed Petri dishes with watch-glasses containing a few cubic centimetres of various liquids inside:

$\begin{array}{cccc}\text { P.d.'s with } & \text { P.d.'s with } & \text { P.d.'s with } & \text { P.d.'s with } \\ \text { nothing inside. } & \text { water. } & \mathrm{I} \% \mathrm{NaOH} . & \mathrm{I} \% \mathrm{H}_{2} \mathrm{SO}_{4} \text {. } \\ 5.69 & 5.87 & 5.77 & 6.5^{2}\end{array}$

Summarizing the results of all the experiments made with potato agar as medium on the lines indicated in Table $\mathrm{X}$, one can draw the following general conclusions :

Experiments in containers.-The amounts of growth over water and over I per cent. sulphuric acid are not appreciably different, as the staling factor is very much reduced by simple dilution due to the large airspace introduced. Both are distinctly greater than the growth in closed Petri dishes and in the open Petri dishes over soda. Of the last two, one was sometimes the greater, sometimes the other, and no general rule was found. The growth in the containers over water in 5 per cent. carbon dioxide was always the best.

Experiments in closed Petri dishes.--Here the small amount of water introduced is of no value as it rapidly becomes alkaline: the improvement due to the introduction of sulphuric acid is here rendered distinct.

Experiments similar to those described for Sphaeropsis were carried out at the same time for Fusarium. The results here were of the same type, and all the results established for Sphaeropsis found a parallel in the experiments with Fusarium. The results were, however, somewhat different in degree. The most striking difference lay in the fact that while 
one could always get distinct improvement in Sphaeropsis cultures by simply increasing the volume of free atmosphere over the cultures, i. e. by growing the cultures in large containers, the corresponding effect with Fusarium was in many cases only slight. This indicates that the ammonia factor is relatively less important in the staling of Fusarium than in that of Sphaeropsis. On the other hand, the effects produced by a moderate concentration of carbon dioxide are in every respect as marked with Fusarium as in the other case.

An experiment with Colletotrichum Lindemuthianum indicated that its behaviour was very similar to that of Fusarium.

On examining the results given in Table $\mathrm{X}$ one sees that the general result can be expressed in the following form :

(a) The growth of Sphaeropsis (on a medium from which ammonia is evolved) is improved by any treatment which causes dilution of the ammonia or its removal.

(b) The growth of the same fungus is improved by allowing the accumulation (within limits) of the carbon dioxide of respiration. Over soda, which keeps the carbon dioxide concentration down to practically zero, growth is least ; over water, where under the experimental conditions a concentration of about 2 per cent. carbon dioxide may be reached, intermediate growth is obtained; in 5 per cent. carbon dioxide the growth is best of all.

To Fusarium statement $(a)$ applies to a less degree, statement (b) equally well.

The only criterion of a staled culture so far given is based on measurement of the diameter of the mycelium at stated intervals. Staling begins when the daily rate of growth begins to diminish. Staling, however, brings on certain characteristic appearances in a fungal colony, so that a staled culture can be recognized as such at a glance.

If the initial inoculum is very sharply localized, the resulting colonies of Sphaeropsis or Fusarium on potato agar are in their earlier stages very nearly perfect circles, and as long as there is no staling at the marginas determined by day-by-day measurements - the colonies remain circular. Also the various controls are extremely uniform in their rate of growth. Once staling at the margin appears, the colonies tend to depart from the circular outline, the margin becoming wavy or indented. Such a sinuous margin is in the writer's experience an invariable indication of staling, at least in the two fungi here dealt with.

The staled cultures further begin to show considerable variation in their rate of spread, so that the variation between the individual cultures becomes much greater. This variability is much greater as a rule in plates where the medium is shallow than where it is deep. Shallowness of medium in many cases accelerates the incidence of staling, and therefore the small differences in depth which occur from plate to plate and from 
one part to another of the same plate produce a marked effect on the growth.

Another striking contrast between staled and unstaled cultures of the two fungi in question is illustrated in Fig. 7, which represents diagrammatically a section in elevation of two cultures of either fungus, the first unstaled and the second staled. In the unstaled culture the height of the 'surface' of the aerial mycelium slopes down gradually to the growing margin, which latter may have a more or less broad fringe with practically no aerial mycelium. The margin of a staled culture is as represented in (b) of Fig. 7 . Here the mycelium drops suddenly at the margin, and this feature gets more pronounced as staling advances; finally one may observe even an overhanging margin.

On plates where the medium is poured deep, a feature of staling is a tendency for the submerged portion of the culture to grow slowly past the aerial portion, and thus the diameter of the submerged growth is greater than that of the aerial growth. Cultures showing this appearance are very stale. This suggests that the deeper layers of the plate are slightly less staled than

a.

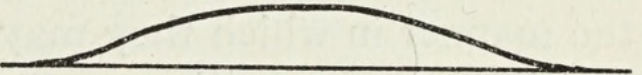

$b$.

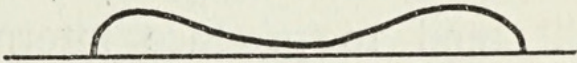

FIG. 7. (Diagrammatic.) Vertical section of unstaled $(a)$ and staled $(b)$ colony. the surface, though why this should be so is not clear. This peculiar effect is not noticeable with shallow media.

A further distinction between a staled and an unstaled colony is to be observed in the nature of the general mycelial growth. In the colonies (Sphaeropsis, Fusarium) growing in closed Petri dishes the mycelium in the central portion of the culture tends to collapse, giving the appearance as if it had been mown. On this central collapsed region Fusarium forms abundant masses of spores, and on the surrounding upstanding aerial mycelium spore masses appear borne aerially, either scattered promiscuously or showing some kind of zonal arrangement. When the culture is grown in 5 per cent. carbon dioxide, where the staling effect is reduced, collapse of the central mycelium is much delayed, and there is everywhere a greatly reduced tendency to sporulation. The exact relationship between sporulation and staling is probably very complex, but there would appear to be every probability that, if the dynamics of the staling process were more completely investigated, considerable light would be thrown on the problem of sporulation.

The same appearances are shown by Sphaeropsis cultures, with the exception that no spores appeared in any case, at least during the limited duration of each experiment. Here it is only necessary to compare the type of growth in a closed Petri dish with that in an open one (in a large 
container) in order to see in the former all the distinct morphological features associated with staling, while the latter shows none of them.

The question of the intermingling or non-intermingling of two fungal colonies (of the same or of different species) is intimately connected with the question of staling. The only physiological interpretation of the nonintermingling of two colonies is that by the combined action of the two colonies the zone of medium immediately between them becomes so stale to both fungi that neither of them can cross it. The phenomenon is not one of absolute incompatibility, and investigation would probably show that any fungus could be made to intermingle with any other under appropriate conditions. In the case of species which have an intense staling action, it would, of course, be much more difficult to find such conditions than with species which have only a feeble staling activity. The question is obviously one of the chemical nature of the various staling products and of the manner in which they may be rendered ineffective.

That intermingling or the converse is a relative matter can be easily demonstrated. If two colonies fail to intermingle under a given set of circumstances, any alteration of the latter which tends to reduce staling will tend to produce intermingling. Thus, if two colonies planted at a certain distance apart fail to meet, they can be made to intermingle by reducing sufficiently the distance between the two inocula. Also, while two colonies may meet when sown at a certain distance apart on a deep medium, they may fail to do so when grown under like conditions on a shallow layer of the same medium. Again, intermingling or non-intermingling can be determined by simply manipulating some of the staling factors, e.g. the gaseous factors discussed in the present paper. Table XI illustrates an experiment with Sphaeropsis along these lines. The top row of figures in the table represents the number of days from the start of the experiment; the figures below represent the shortest distance from the margin of one colony to that of the other; the medium was potato agar in Petri dishes.

\section{TABLE XI.}

$\begin{array}{ccccccccc}\text { Closed dishes in air: } & 0 . & 5 . & 7 . & 8 . & 9 . & \text { I 2. } & \text { I5. } & \text { I 7. } \\ \text { Deep medium } & 7.0 & \mathrm{I} \cdot 7 & \text { met } & - & - & - & - & - \\ \text { Shallow medium } & 7.0 & 2.3 & \mathrm{I} \cdot 4 & - & 0.8 & 0.8 & 0.8 & 0.75\end{array}$

Open dishes in container in air :

Deep medium

Shallow medium

7.0

$1 \cdot 25$

met

0.25

$\overline{\text { met }} \quad-$

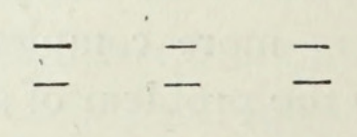

Open dishes in container in $7.5 \% \mathrm{CO}_{2}$ :

Deep medium

Shallow medium
$7 \cdot 0$
$7 \cdot 0$

$\mathrm{I} \cdot \mathrm{I}$
$\mathrm{I} \cdot 4$

met met 


\section{General Discussion.}

In the earlier portion of this paper the growth-rate curves of a number of fungi were given, from which it appeared that after an initial period of slow growth the rate of growth reached a maximum at which it remained steady, or from which it subsequently declined. This effect of reduced rate of growth is obviously a case of staling of the growing margin of the mycelium by the products formed within its mass, a conclusion with which all the results of the present paper are in agreement. These products either diffuse outwards beyond the limits of the growing margin or, as in the case of ammonia, pass into the atmosphere of the culture and from there are absorbed by the cultural medium. The result of this is that the portion of the medium on which the growing margin of the colony is entering becomes less suitable day by day for the growth of the fungus, and thus staling of the margin begins.

In any case where staling does not occur (e.g. Botrytis growing on potato agar), one must assume that the fungus produces no appreciable amount of staling substances or that it is very insensitive to their presence, whereas in the case of a staling culture, e.g. Fusarium on potato agar, the assumption must be either that the fungus produces large quantities of staling substances or that it is highly sensitive to their action. Now, though the ultimate proof must be a chemical one, there is little doubt that it is the former alternative that rules. Botrytis is a non-staling form simply because it produces relatively little staling substance. In both cases the dominant feature of the staling process from the chemical point of view is the development of an alkaline reaction in the potato medium, and this is very much more pronounced with Fusarium than with Botrytis. Again, it is easy to show that the sensitiveness of Botrytis to alkalinity in the medium is much greater than that of Fusarium. (See Table V.)

The views here put forward are strikingly confirmed by a comparison of the behaviour of colonies of the two organisms growing on the same plate, as compared with controls in which each is growing alone. The growth of Fusarium in presence or in absence of Botrytis is very much the same: that of Botrytis is obviously affected by the presence of the Fusarium. On such a medium as potato agar where ammonia is evolved by the Fusarium, the growth of the Botrytis colony is reduced and finally stopped in all directions. On a medium from which ammonia is not evolved, the Botrytis colony is strongly staled on the side next the Fusarium, so that it appears excentric, while the Fusarium colony will preserve its circular outline much longer.

The only view which will thus meet the experimental facts is that Botrytis tends to be a fungus of unlimited growth simply because its capacity to form staling products is small, whereas Fusarium, though it is compara- 
tively tolerant of staling substances, nevertheless produces them in such quantity that its growth tends to be limited.

Though the amount of staling shown by a fungus depends on the experimental conditions, and especially on the nature of the medium on which it is grown, yet it would appear that a fungus which shows staling on one medium is likely to show a tendency in the same direction on any other medium, and thus one could speak in a general way of Fusarium as being of the staling, and Botrytis as being of the non-staling, type.

In interpreting the growth-curves of the same fungus when grown under different experimental conditions, one has to take account of the amount of mycelium within the body of the colony, and it is in respect of this point that the difficulty of this method of experiment lies. However, certain broad conclusions can be drawn. Take, for instance, the growth of Sphaeropsis on potato agar in a closed Petri dish as compared with that in an open one, all other conditions being the same. Here the mycelia under the two conditions are identical in all appearances in the early stages. The differences in amount of staling which appear later cannot be due to different rates of mycelium production, as the amount of mycelium present at the time of incidence of staling is obviously the same in the two cases. In fact, the non-staling culture has probably much more mycelium after a time than the staled one, and thus the unstaled type of growth is maintained in spite of what is probably a greater evolution of the staling substances. The only interpretation which will suit the facts is that the staled growth in the one case results from the accumulation of an active staling substance, an accumulation which is kept in abeyance in the other by the experimental arrangements.

The more staled growth that one finds on certain media when present in a shallow as compared with a deep layer, is to be interpreted somewhat differently. Here it is obvious from inspection that there is less mycelial growth at all stages on the shallow layer of medium. In this case the interpretation must be that though a greater amount of staling substances is formed on the deep layer in accordance with the greater amount of mycelium present, nevertheless it is not so effective in producing staling at first as it is free to diffuse away into a larger volume of medium. The results of growth measurements on deep and shallow layers of medium depend very markedly on the nature, and especially on the concentration, of the nutrient medium, and this will be dealt with more fully in a subsequent paper.

Throughout this paper we have dealt merely with one factor of staling, viz. ammonia. This, however, is not the only factor, or even the only alkaline factor. Thus it is easy to show that a medium on which Fusarium or Sphaeropsis has been grown with evolution of ammonia is still alkaline, even after all the ammonia has been driven off. There is thus a formation of fixed alkali which is more marked in the case of Fusarium than of 


\section{Fungi on Culture Media.}

Sphaeropsis. In the experiments recorded in this paper it was shown that the improvement of growth resulting from diminution of the ammonia factor was more marked in the case of Sphaeropsis than of Fusarium. In the latter case mere removal of ammonia did not very greatly improve growth, but raising of the carbon dioxide concentration in the atmosphere of the colony produced a very distinct effect. Thus we may say that in the case of Sphaeropsis the volatile alkali is more effective in causing staling than the fixed alkali, whereas with Fusarium the converse is true.

It will be observed that the points brought forward in the present paper explain fully the unexpected results mentioned in the introduction. The fact that some fungi (e.g. Fusarium, Sphaeropsis) show more growth after a time in a moderate concentratian of carbon dioxide than in air is correlated with the production of an alkaline staling reaction in the medium. Thus, though carbon dioxide at the concentrations employed (Io to 20 per cent.) cannot be looked upon in any other sense than as a retarder of growth, it produces the opposite effect in such cases in virtue of its neutralizing action on the staling products. With such fungi as Botrytis cinerea, where production of alkali is very slight, the effect described does not appear.

\section{SUMMARY.}

Curves of the rate of growth, as measured in terms of the diameter of the colony, are given for a number of fungi. The general feature of these curves is that in the early stages the rate of growth is small, and that it then rises to a maximum which may, or may not, be maintained.

Fungi which keep up this limiting rate of growth are described as being of the non-staling type ; those in which the rate of growth falls off from the maximum.are described as being of the staling type.

The growth of Sphaeropsis malorum and of Fusarium sp. on potato agar which is of the staling type is studied in detail, when it is found that the relative amount of staling can be modified by various modifications of the experimental conditions, by varying the depth of the medium, and in particular by the arrangements made for disposing of two volatile products of the metabolism of the fungus, carbon dioxide and ammonia. According as the one or the other is allowed to accumulate, a greater or less degree of staling will result.

The amounts of ammonia formed in Sphaeropsis cultures on a number of media are determined.

Correlations between staling and sporulation, and between staling and the phenomena of intermingling, are indicated. 


\section{$2 \mathrm{BHL}$ Biodiversity Heritage Library}

Brown, William. 1923. "Experiments on the growth of fungi on culture media." Annals of botany 37, 105-129.

https://doi.org/10.1093/oxfordjournals.aob.a089828.

View This Item Online: https://www.biodiversitylibrary.org/item/270686

DOI: https://doi.org/10.1093/oxfordjournals.aob.a089828

Permalink: https://www.biodiversitylibrary.org/partpdf/319071

\section{Holding Institution}

New York Botanical Garden, LuEsther T. Mertz Library

\section{Sponsored by}

BHL-SIL-FEDLINK

\section{Copyright \& Reuse}

Copyright Status: Public domain. The BHL considers that this work is no longer under copyright protection.

This document was created from content at the Biodiversity Heritage Library, the world's largest open access digital library for biodiversity literature and archives. Visit BHL at https://www.biodiversitylibrary.org. 